\title{
Prevalence of Tissue $B R C A$ Gene Mutation in Ovarian, Fallopian Tube, and Primary Peritoneal Cancers: A Multi-Institutional Study
}

\author{
Arb-Aroon Lertkhachonsuk ${ }^{1}$, Prapaporn Suprasert ${ }^{2 *}$,Tarinee Manchana ${ }^{3}$, \\ Thannaporn Kittisiam ${ }^{4}$, Nuttavut Kantathavorn ${ }^{5,6}$, Tharintorn Chansoon, \\ Surapan Khunamornpong ${ }^{8}$, Natkrita Pohthipornthawat ${ }^{9}$, Siriwan Tangjitgamol ${ }^{10}$, \\ Taksa Luasiripanthu ${ }^{6}$, Chinachote Teerapakpinyo ${ }^{11}$, Shanop Shuangshoti ${ }^{12}$, \\ Nareenart Iemwimangsa ${ }^{13}$,Wasun Chantratita ${ }^{13}$
}

\begin{abstract}
Background and objective: Ovarian, fallopian tube, or primary peritoneal cancer patients with $B R C A$ gene mutation have enhanced sensitivity to platinum-based regimens and PARP inhibitors. However, the knowledge regarding $B R C A$ mutation in Thai patients is limited. This study aimed at identifying the prevalence and characteristics of somatic and germline $B R C A 1$ and 2 mutations in Thai patients with these cancers. Materials and Methods: The paraffin blocks of tumors with histology of high grade serous, high grade endometrioid, or clear cell carcinoma obtained between June 2016 and December 2017 were analyzedto evaluate $B R C A$ mutation using next-generation sequencing system. Blood or normal tissue paraffin blocks of positive patients were further tested for germline $B R C A$ mutation. Results: Tissue paraffin blocks of 178 patients were collected but only 139 were analyzed. Positive $B R C A$ mutation was identified in 24 patients (17.3\%): BRCA1 in 13 cases, $B R C A 2$ in 10 cases, and $B R C A 1$ and 2 in the rest one. Germline mutation study in blood or normal tissue in 23 positive patients revealed $B R C A$ mutation in 14 cases, BRCA1 in 8 cases and $B R C A 2$ in 6 cases. Overall, the prevalence of somatic and germline mutation was $6.5 \%$ ( 9 out of 138 patients) and $8.7 \%$ (14 out of 138 patients), respectively. The most common histology associated with $B R C A$ mutation was high grade serous cancer (27.3\%). No significant difference was found between patients with or without $B R C A$ mutation in terms of stage, outcome, platinum status, and survival outcome. Conclusion: $B R C A$ mutation was demonstrated in less than $10 \%$ of Thai ovarian cancer patients. Higher rate of mutation was found in high grade serous cancer.
\end{abstract}

Keywords: $B R C A$ mutation- ovarian cancer- fallopian tube cancer- peritoneal cancer

Asian Pac J Cancer Prev, 21 (8), 2381-2388

\section{Introduction}

Epithelial ovarian cancer (EOC) is the 6th most common cancer among Thai females with the age standardized rate of 6.0 per 100,000 females (Wilailak and Lertchaipattanakul, 2016; Torre et al., 2018).

Among prognostic factors affecting survival, stage of the disease is the most important one. For instance,

\footnotetext{
${ }^{1}$ Division of Gynecologic Oncology, Department of Obstetrics and Gynaecology, Faculty of Medicine, Ramathibody Hospital, Mahidol University Bangkok, Thailand. ${ }^{2}$ Division of Gynecologic Oncology, Department of Obstetrics and Gynecology, Faculty of Medicine, Chiang Mai University, Chiang Mai, Thailand. ${ }^{3}$ Division of Gynecologic Oncology, Department of Obstetrics and Gynecology, Faculty of Medicine, Chulalongkorn University and King Chulalongkorn Memorial Hospital, Bangkok, Thailand. ${ }^{4}$ Division of Gynecologic Oncology, Department of Obstetrics and Gynecology. Faculty of Medicine Vajira Hospital, Navamindradhiraj University, Bangkok, Thailand. ${ }^{5}$ Faculty of Medicine and Public Health, HRH Princess Chulabhorn College of Medical Science, Chulabhorn Royal Academy, Bangkok, Thailand. ${ }^{6}$ Chulabhorn Hospital, HRH Princess Chulabhorn College of Medical Science, Chulabhorn Royal Academy, Bangkok, Thailand. ${ }^{7}$ Department of Pathology, Faculty of Medicine, Ramathibodi Hospital, Mahidol University, Bangkok, Thailand. ${ }^{8}$ Department of Pathology, Faculty of Medicine, Chiang Mai University, Chiang Mai, Thailand. ${ }^{9}$ Division of Gynecologic Pathology and Cytology, Department of Obstetrics and Gynecology, Faculty of Medicine, Chulalongkorn University and King Chulalongkorn Memorial Hospital, Bangkok, Thailand. ${ }^{10}$ Department of

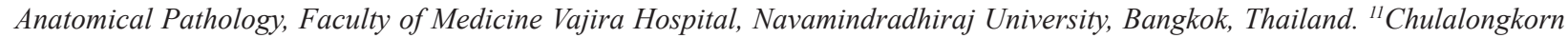
GenePRO Center, Department of Pathology, Faculty of Medicine, Chulalongkorn University, Bangkok, Thailand. ${ }^{12}$ Department of Pathology, Faculty of Medicine, Chulalongkorn University, Bangkok, Thailand. ${ }^{13}$ Center for Medical Genomics, Faculty of Medicine Ramathibodi Hospital, Mahidol University, Bangkok, Thailand. *For Correspondence: psuprase@gmail.com
} 
2-year survival rate ranged from $90 \%$ in early and $40-50 \%$ in advanced stages (Webber and Friedlander, 2017). Another important prognostic factor is the response to first-line chemotherapy or platinum-sensitivity status. When the recurrences occur either later than 6 months or sooner after the last therapy, the diseases are regarded as platinum-sensitive or platinum-resistant, respectively. Platinum-based chemotherapy is usually used for platinum-sensitive disease; whereas, other non-platinum chemotherapies can be the option for platinum-resistant diseases (Jansaka and Suprasert, 2014; Webber and Friedlander, 2017).

Unfortunately, the patients with platinum-sensitive disease, who have responded to platinum-based chemotherapy re-induction, may have subsequent recurrences. The second-relapse generally have lower responses to treatment with platinum or other chemotherapeutic drugs (Jansaka and Suprasert, 2014; Webber and Friedlander, 2017). Hence, maintenance therapy after primary treatment has emerged in order to extend the progression-free period and survival.

Among drugs that are used as maintenance therapy, poly (adenosine diphosphate [ADP]) ribose polymerase (PARP) inhibitor (PARPi) is a recent efficient agent. PARP is an enzyme that repairs the single-strand break DNA in tumor cells. The mechanism of PARPi action is through inhibiting this enzyme, preventing DNA repair, and leading to further damage to the other DNA strand or double-strand break DNA. The double-strand break DNA can be repaired to normal via homologous recombinant pathway by $B R C A 1 / 2$ gene. In patients with $B R C A 1 / 2$ mutation, the repair mechanism cannot take place leading to cell death eventually (Hennessy et al., 2010; Ledermann et al., 2014; Pujade-Lauraine et al.,2017). Evidence-based data from previous studies showed that PARPi could prolong remission in patients especially those with platinum-sensitive recurrent ovarian cancer. This benefit of PARPi maintenance therapy on progression-free survival was evidenced especially in patients with either germline or somatic BRCA1/2 mutation (Hennessy et al., 2010; Cancer Genome Atlas Research Network 2011; Ledermann et al., 2014; Pujade-Lauraine et al., 2017). Hence, knowing the status of BRCA1/2 mutation in ovarian cancer patients is important in order to support a decision a clinician for selecting an appropriate treatment option for the patients.

The prevalence of germline BRCA1/2 mutation, according to previous reports, ranged from 14 to $29 \%$ in Asia (Wu et al., 2017; Enomoto et al., 2019; Kwon et al., 2019). Higher percentages of mutation were found in some types of EOC, such as high grade serous cancer, which was found to have BRCA1/2 mutation as high as $28.5 \%$ (Kwon et al., 2019).

With the possible ethnic influence, the prevalence of $B R C A 1 / 2$ mutation may differ across different countries. The genetic test, including testing of $B R C A 1 / 2$ mutation, in ovarian cancer patients had not been a standard practice in our country. Hence, the prevalence of $B R C A 1 / 2$ gene mutation in our population remained unknown. The Thai Gynecologic Cancer Society is aware of the increasing role of PARPi in treatment of ovarian cancer, so this study was conducted among tertiary centers for cancer care in Thailand to determine the $B R C A 1 / 2$ mutation status in Thai patients with EOC.

\section{Materials and Methods}

The study was approved by local ethic committees of each participating institution. Inclusion criteria were as follows: Thai patients who had EOC, fallopian tube cancer, and primary peritoneal adenocarcinoma (PPA), operation between June 1, 2016 and December 31, 2017, histopathology of high grade serous, high grade endometrioid, or clear cell adenocarcinoma, available paraffin blocks for tissue processing, and available clinical data. In any cases of mixed cell types, these aforementioned histopathologic components must be at least $10 \%$ for the pathological processing and examination.

\section{Laboratory data}

To confirm tumor histopathology and grade, the representative slides containing tumor from each institution were submitted for central pathologic review. The central pathologic review was done by a gynecologic pathologist (S.K.). The paraffin blocks of tumors were then randomly submitted to one of the two qualified genomic laboratories: 1) Center for Medical Genomics, Faculty of Medicine, Ramathibodi Hospital and 2) Chula GenePRO Center, Faculty of Medicine, King Chulalongkorn Memorial Hospital. Tumor cells percentage was reviewed by the pathologists (S.S). A cross-validation of the whole process from DNA extraction through data interpretation between both laboratories was executed in 10 samples before proceeding with the $B R C A 1$ and $B R C A 2$ mutation testing in the remaining cases. which were equally distributed to both laboratories.

DNA extraction and quality assessment were performed using QIAamp DNA FFPE Tissue kit and GeneRead DNA QuantiMIZE kits according to manufacturer's recommendation (Qiagen, Hilden, Germany). BRCA1 and $B R C A 2$ mutations were analyzed using GeneReader nextgeneration sequencing (NGS) system (Qiagen, Hilden, Germany). Quality of data sequencing was examined using unique molecular index (UMI) coverage, including $100 \mathrm{X}>90 \%$ and $60 \mathrm{X}>95 \%$ of $B R C A 1$ and $B R C A 2$ coding regions. The samples that showed inadequate DNA quality or UMI coverage were discarded to avoid false positive and false negative results.

The variant classification was preliminarily evaluated by Ingenuity Variant Analysis (Qiagen) and manually reviewed according to the American College of Medical Genetics and Genomics (ACMG)/ the Association for Molecular Pathology (AMP) guidelines (Richards et al., 2015; Li et al., 2017). The final result was made after discussion and mutual agreement between both laboratories in all specimens. The patients, who had tissue $B R C A 1 / 2$ mutation, were counselled for germline testing. For germline testing, 5 milliliters of blood were drawn from patients, who had agreed and signed inform consent, using bidirectional direct sequencing. For other patients, the genetic testing was performed using normal tissue taken from the paraffin blocks. 
The prevalence of tissue $B R C A$ mutation was calculated by dividing the number of patients who had $B R C A$ mutation from tumor tissue to total number of patients who had tumor tissue testing. On the other hand, the prevalence of germline or somatic $B R C A$ mutation was calculated different. This number would be divided by the total number of patients who had tumor tissue testing but excluded those patients with tissue $B R C A$ mutation whose peripheral blood or the paraffin blocks with normal tissue were unavailable.

\section{Clinical Data}

Clinical data of the patients extracted from their medical records. These data included age, stage of cancer according to the International Federation of Gynecology and Obstetrics (FIGO 2014), histopathology, dates of treatments, outcomes, status of disease, and living status. The patients who developed progression during the course of chemotherapy or had recurrence within 6 months after the completion of chemotherapy were classified as platinum-refractory and platinum-resistant, respectively. or else were classified as platinum-sensitive. Progression-free survival (PFS) was calculated from the date on surgery or the date of start chemotherapy in patients who underwent neoadjuvant chemotherapy until the date of progression, recurrence, or last evaluation. Overall survival (OS) was obtained from the same date that using for calculated PFS to date of death or last follow-up in patients who were alive at the end of the study.

\section{Sample Size Calculation}

A target minimum sample size was calculated according to the previous publication reporting $10 \%$ prevalence of BRCA1/2 mutation in EOC (Zhao et al., 2017). With respect to alpha error at $<0.05$ added with $10 \%$ loss of data, 160 patients were needed to be included in the study.

\section{Statistical Analysis}

Statistical analysis of the data was carried out using IBM SPSS statistics for Windows program (version 22; IBM Corporation, Armonk, NY, USA). Descriptive data were presented as number and percentages, mean with SD or median with range. Patients with BRAC 1/ 2 mutation or without BRAC 1/ 2 mutation were compared using Chi-square or Fisher's Exact test as appropriate. Survival data were analyzed with Kaplan Meier and compared between groups with log-rank test. A p-value of $<0.05$ was considered as statistical significance.

\section{Results}

A total of 178 patients from 5 cancer institutions were included in this study. the instiutions were Faculty of Medicine Ramathibodi Hospital, Mahidol University $(\mathrm{n}=52)$, Chiang Mai University Hospital, Chiang Mai University $(\mathrm{n}=44)$, King Chulalongkorn Memorial Hospital, Chulalongkorn University $(n=43)$, Faculty of Medicine and Vajira Hospital, Navamindradhiraj University $(\mathrm{n}=31)$, and Chulabhorn Hospital, Chulabhorn
Royal Academy $(n=8)$. All hospitals were in Bangkok, except for Chiang Mai University Hospital which is in the northern part of Thailand.

The mean age of the patients was $56.9 \pm 11.4$ years. Ovarian cancer was the most common diagnosis among the included patients $(86.5 \%)$, followed by fallopian tube cancer, $(9.6 \%)$ and primary peritoneal cancer $(3.9 \%)$. About $57.4 \%$ of the patients were in advanced stage of the disease. High grade serous cancer was the most common histopathology (56.7\%). Approximately $11.2 \%$ of patients had neoadjuvant chemotherapy prior to surgery; whereas,

Table 1. Clinico-Pathologic Data of the Patietns and Tumors $(\mathrm{N}=178)$

\begin{tabular}{lc}
\hline Clinico-pathological features & $\mathrm{N}(\%)$ \\
\hline Diagnosis & \\
CA Ovary & $154(86.5)$ \\
CA tube & $17(9.6)$ \\
PPA & $7(3.9)$ \\
Histology & \\
Serous & $101(56.7)$ \\
Clear cell & $69(38.8)$ \\
Endometrioid & $4(2.2)$ \\
Mixed & $4(2.2)$ \\
Stage & \\
I & $52(29.2)$ \\
II & $24(13.5)$ \\
III & $80(44.9)$ \\
IV & $22(12.4)$ \\
Neoadjuvant chemotherapy & $20(11.2)$ \\
Adjuvant chemotherapy & $171(96.1)$ \\
Regimen & \\
Carboplatin + paclitaxel & \\
Carboplatin & $121(68.0)$ \\
Carboplatin + Pegylated liposomal doxorubicin & $37(49.5(20.8)$ \\
Carboplatin + paclitaxel+Bevacizumab & $1(0.6)$ \\
Outcome after primary chemotherapy treatment Responses \\
Complete response & $118(66.3)$ \\
Partial response & $20(11.2)$ \\
Stable disease & $4(2.2)$ \\
Progression & $26(14.6)$ \\
Missing data & $10(5.6)$ \\
Recurrence & $18(5.6)$ \\
Progression after response to chemotherapy stative & $23(12.9)$ \\
\hline
\end{tabular}

Asian Pacific Journal of Cancer Prevention, Vol 212383 
Table 2. BRCA Gene Mutation According to the Histology $(\mathrm{N}=139)$

\begin{tabular}{|c|c|c|c|c|c|c|c|}
\hline \multirow[t]{3}{*}{ Histology } & \multicolumn{7}{|c|}{ BRCA result (\%) } \\
\hline & \multicolumn{4}{|c|}{ Positive } & \multirow[t]{2}{*}{ Negative } & \multirow[t]{2}{*}{ VUS } & \multirow[t]{2}{*}{ Tota } \\
\hline & BRCA1 & $B R C A 2$ & $B R C A 1 \& 2$ & Total & & & \\
\hline Serous & $13(16.9)$ & $7(9.1)$ & $1(1.3)$ & $21(27.3)$ & $49(63.6)$ & $7(9.1)$ & 77 \\
\hline Clear & - & $3(5.5)$ & - & $3(5.5)$ & $48(87.3)$ & $4(7.3)$ & 55 \\
\hline Endometrioid & - & - & - & - & $4(100)$ & - & 4 \\
\hline Mixed & - & - & - & - & $3(100)$ & - & 3 \\
\hline Total & $13(9.4)$ & $10(7.2)$ & $1(0.7)$ & $24(17.3)$ & $104(74.8)$ & $11(7.9)$ & 139 \\
\hline
\end{tabular}

VUS,Variant of Uncertain significance; Mixed histology included endometrioid caecinoma + clear cell carcinoma (2 cases), high grade serous carcinoma + celar cell carcinoma ( 1 case)

almost all (96.1\%) received adjuvant chemotherapy. The most common first-line chemotherapy was carboplatin plus paclitaxel (68.0\%). Complete response after primary treatment was achieved in 118 patients $(66.3 \%)$ and 55 patients $(30.9 \%)$ had later developed recurrence.

Regarding $B R C A$ results, there were only 139 patients whose paraffin-blocks could be further processed for the test. The others were disapproved due to suboptimal quality of DNA and sequencing data, so were excluded from further investigation. Out of 139 patients, the tissue $B R C A$ gene mutation was found in 24 cases (17.3\%): $B R C A 1$ mutation in 13 cases $(9.5 \%), B R C A 2$ mutation in $10(7.2 \%)$, and both $B R C A 1$ and 2 mutation in 1 case $(0.7 \%)$. The remaining 11 patients $(7.9 \%)$ were reported to have variants of uncertain significance (VUS).

Table 1 shows BRCA gene mutation according to the histology. Only high grade serous cancer and clear cell carcinoma showed positive $B R C A$ gene mutation: high grade serous cancer in 21 from 77 patients $(27.3 \%)$ and

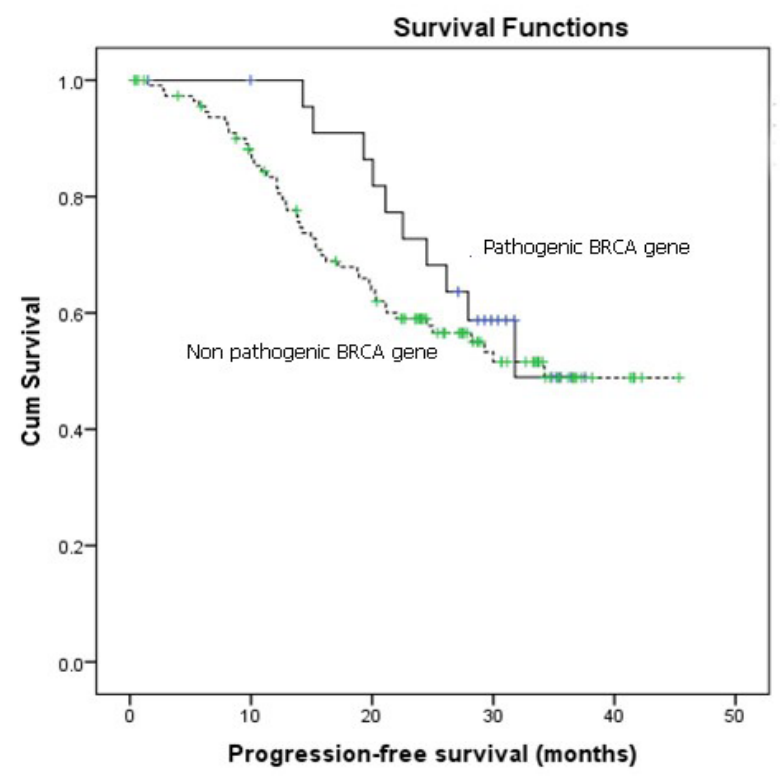

Figure 1. Progression -Free Survival in Patients with and without Pathogenic BRCA Gene. Median progression free survival: Pathogenic BRCA gene $=31.8$ months vs. non-pathogenic BRCA gene = 30 months; 2 year progression free survival : Pathogenic BRCA gene = $68.2 \%(95 \%$ CI $48.8-87.6 \%$ ) vs. non- Pathogenic BRCA gene $=59 \%(95 \%$ CI 49.6-68.4); Log rank test $\mathrm{P}=0.43$; $\mathrm{CI}=$ confidence interval clear cell carcinoma in 3 out of 55 ones (5.5\%). Among 24 patients with $B R C A$ positive, all except one could be pursued to have germline $B R C A$ gene mutation testing by using peripheral blood in 18 patients and normal tissue of myometrium from paraffin block in the other 5 patients. The results showed germline positive in 14 patients, 8 $B R C A 1$ mutation and 6 BRCA2 mutation; 13 of which were high grade serous cancer and only 1 was clear cell carcinoma. Thus, among 138 patients who had the data of both germline and somatic, the percentages of germline $B R C A$ gene mutation was $8.7 \%$ (14 out of 138 patients), while the percentage of somatic $B R C A$ gene mutation was $6.5 \%$ (9 in 138 cases). There were $4 B R C A 1$ mutation, 4 $B R C A 2$ mutation, and $1 B R C A 1$ and 2 mutation. The patients who had germline mutation was significantly younger than those with only somatic mutation (48.4 \pm 10.6 versus $61.6 \pm 12.9$ years, $\mathrm{P}=0.014)$. Thus, at least the prevalence rates of germline and somatic $B R C A$ mutation in our study were $8.7 \%$ and $6.5 \%$, respectively.

Table 2 summarizes findings on $B R C A$ gene mutation identified by next generation sequencing. Three mutation patterns were identified, including 11 frameshifts, 9 nonsenses, and 2 missenses. Missense mutations were

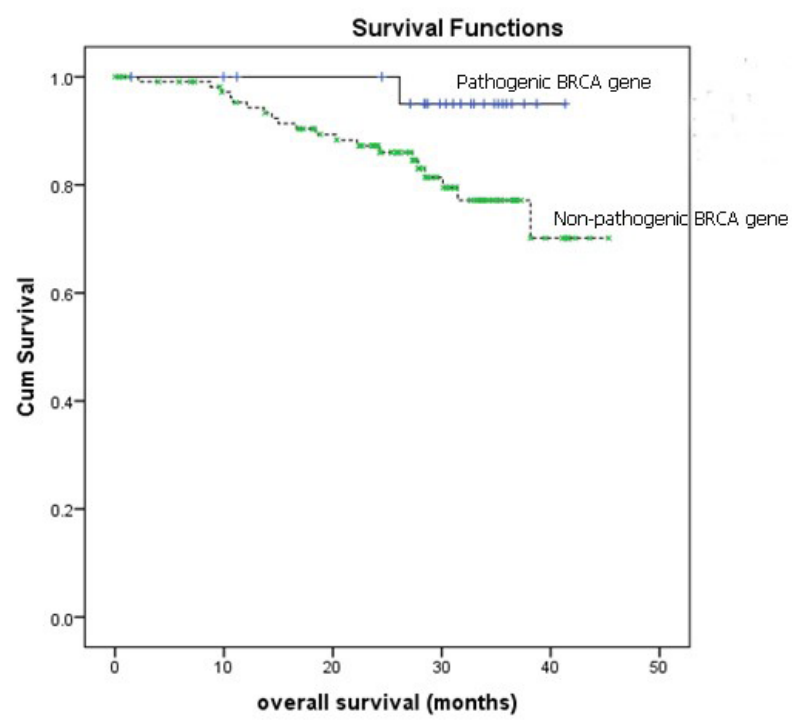

Figure 2. Overall Survival (OS) in Patients with and without Pathogenic $B R C A$ Gene. Log rank test $\mathrm{P}=0.077$ 2-yrs overall survival : non-pathogenic BRCA gene $=$ $87.2 \%(95 \%$ CI $88.8-100 \%)$, pathogenic BRCA gene $=$ $95 \%$ (95\% CI 77.6-96.8\%); CI = confidence interval. 


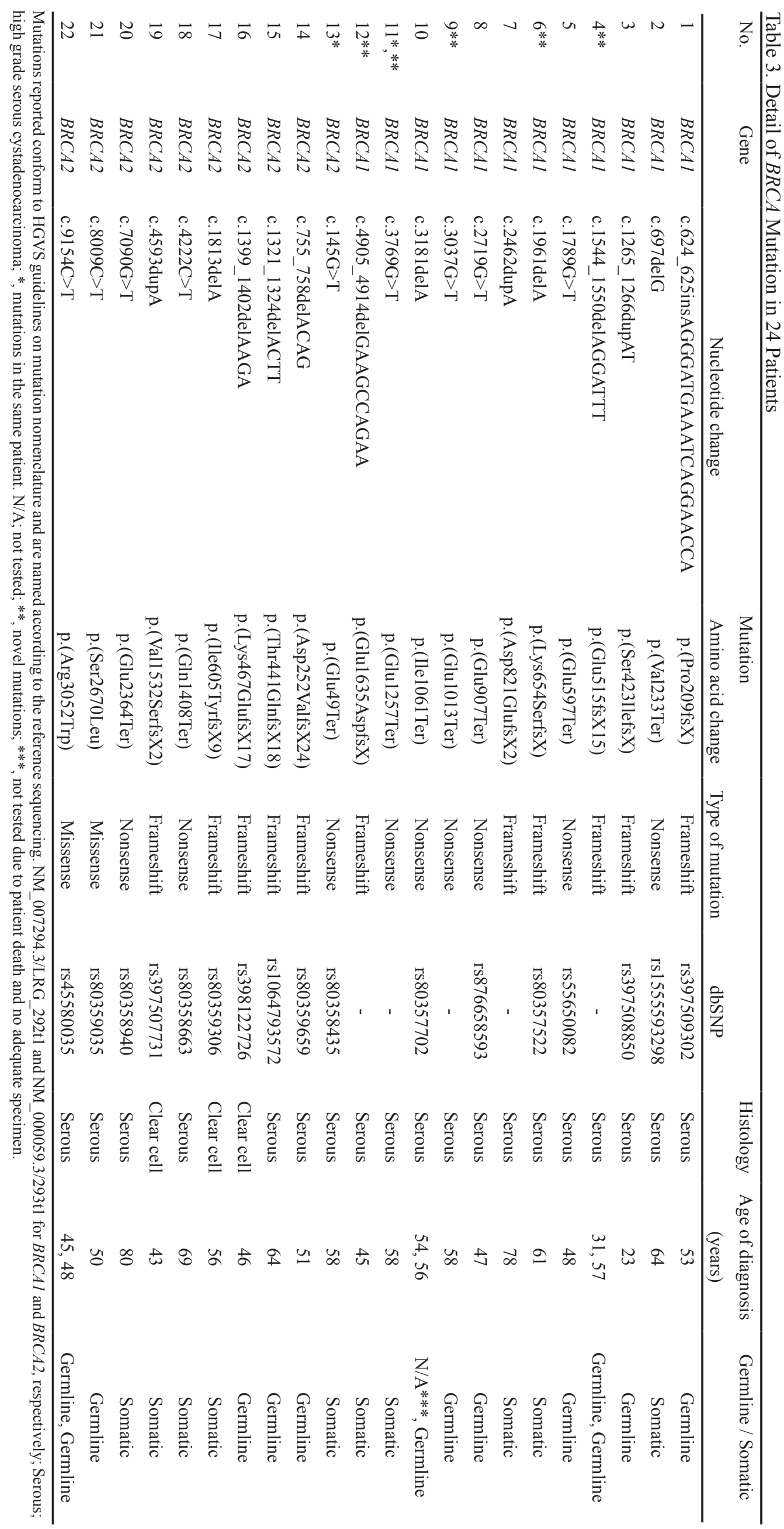


Table 4. Clinical Data of the Patients According to $B R C A$ Results $(\mathrm{N}=139)$

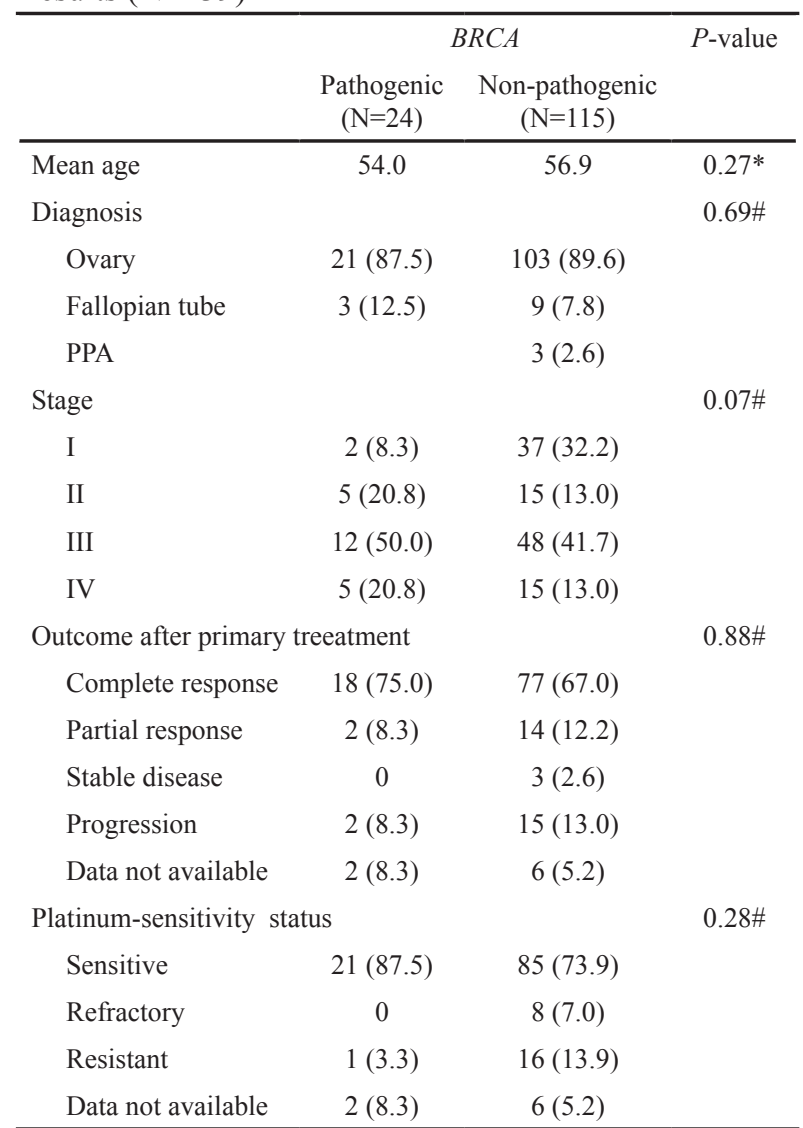

Non-pathogenic, $B R C A$ negative + VUS (VUS = Variant of Uncertain significance); \#, Fisher Exact test; *, Chi-square test

observed only in $B R C A 2$ genes, while frameshift and nonsense were found in both BRCA1 and BRCA2. Five novel mutations were observed in 6 patients; whereas, the others were known mutations reported in the NCBI database of genetic variation (dbSNP).

This study could not demonstrate any differences between patients with and without tissue $B R C A$ gene mutation regarding age, type of cancer, stage, outcome after primary treatment, and platinum-sensitivity status (Table 3). Figure 1 and Figure 2 also revealed no differences between two groups of patients in terms of 2-year PFS and 2-year OS. However, the OS in patients with pathogenic $B R C A$ gene tended to be better with 2-year OS of $95.0 \%$ (95\% confidence interval $77.6-96.8 \%$ ) compared to $87.2 \%$ (95\% confidence interval $88.8-100 \%)$ in patients without pathogenic $B R C A$ gene ( $\mathrm{P}=0.077)$.

\section{Discussion}

The present study found tissue $B R C A$ mutation in high grade $\mathrm{EOC}$, fallopian tube cancer, and primary peritoneal adenocarcinoma in 24 out of 139 patients (17.3\%). Out of 24 patients, 14 patients revealed germline BRCA 1/2 mutations. Thus, the prevalence of germline and somatic BRCA mutation in our study was at least 10.1\% (14 in 138 cases) and $6.5 \%$ (9 in 138 cases), respectively. These rates corresponded to those reported by a Taiwanese's study. Chao et al., (2016) investigated the occurrence of BRCA $1 / 2$ mutation in 99 formalin-fixed paraffin-embedded tumor samples of tumor and normal tissue obtained from
Taiwanese ovarian cancer patients by using next-generation sequencing. They found BRCA 1 in 7 cases and BRCA 2 in 6 cases, reporting $12.1 \%$ prevalence. In their study, 4 cases (4\%) had somatic mutation. Another study was done in Thailand by Manchana et al., (2019). They investigated the frequency of $B R C A$ mutation in 87 Thai high grade serous cancer and high grade endometrioid ovarian cancer, including the fallopian and peritoneal cancers. They used peripheral blood DNA samplers, DNA extracted from formalin-fixed paraffin embedded blocks (FFPE), or a fresh tumor specimen to analyze $B R C A$ mutation via the next generation sequencing system. The results showed germline $B R C A$ mutation detection in 19 patients $(21.8 \%)$, of which $14(16.1 \%)$ were $B R C A 1$ mutation and $5(5.7 \%)$ were $B R C A 2$ mutation. All positive patients revealed histology as high grade serous cancer. The difference in detection rates of $B R C A$ mutation between our study and Manchanaet al., (2019)'s study was probably due to usage of differed specimens. Our study used only FFPE that might decrease the detection rate due to the variability of tumor samples and intra-tumoral heterogeneity which probably compromised the representativeness of the sample (da Cunha Colombo Bonadio, 2018); whereas, Manchana et al., (2019) used fresh tumor specimen or blood samples aside from FFPE to analyze $B R C A$ prevalence.

The most frequent histology in patients with germline $B R C A$ mutation was high grade serous carcinoma in a range of 5.5-16.7\%, followed by endometrioid carcinoma (4.3-13\%), and clear cell carcinoma (6.3-9.1\%) (Vergote et al.,2016; da Cunha Colombo Bonadio, 2018) . However, in the present study, tissue $B R C A$ mutation was found in $27.3 \%$ of high grade serous carcinoma and $5.5 \%$ of clear cell carcinoma, while none in endometrioid carcinoma. This non-similar outcome might be due to very small number of patients with high grade endometrioid carcinoma in our study. However, BRCA2 mutation was found only in clear cell carcinoma. This finding corresponded to the previous reports suggested that BRCA2 mutation is common in this cell type (Goodheart et al., 2009; Manchana et al., 2019). However, 2 out of 3 patients with clear cell carcinoma had somatic $B R C A 2$ mutation (87.7\%) and only 1 patient (33.3\%) had germline BRCA2 mutation.

Due to different reports on prevalence of $B R C A$ mutation among various histologies, recently the American Society of Clinical Oncology (ASCO) suggest assessing either germline or somatic $B R C A$ gene mutation in all non-mucinous ovarian, fallopian tube, and peritoneal cancers of high grade (Konstantinopoulos et al., 2020). Improvement of treatment outcome is possible through PARPi (Ledermann et al., 2014; Pujade-Lauraine et al., 2017; da Cunha Colombo Bonadio et al., 2018; Hennessy et al., 2010; Dougherty et al., 2017). Gori et al., (2019) summarized an initial step of $B R C A$ mutation gene investigation by studying $B R C A$ 1/2 mutation on tumor tissue. The test for identifying spectrum of genomic rearrangements (i.e., deletions or duplications of one or more exons, or of the whole gene) yielded high sensitivity. In the patients, whose tissues were positive for $B R C A 1 / 2$ mutation, genetic counseling and further investigation for 
germline mutation from their peripheral blood should be deferred as the next step of investigation. If the blood test be positive, these patients will benefit from PARPi therapy and should be under close surveillance for a possible development of second malignancy.

The major problem with using tissue sample for testing pathogenic gene was the quality of FFPE clinical specimens. Although next generation sequencing used in this study can sequence entire $B R C A 1 / 2$ coding regions, epigenetic and structural changes could not be detected in this study. Thirty nine DNA samples $(21.9 \%)$ were unable to obtain $B R C A$ mutation status due to insufficient DNA quality. Various factors affect FFPE DNA quality in molecular genomics test, such as tissue fixation time, storage time, and archive temperature (Einaga et al., 2017). In this study, we selected FFPE tissues blocks during June 2016 to December 2017 and finished the DNA extraction within 2018 ( $<3$ years) to avoid long-term storage effect. Therefore, fixation time and archive temperature could lead to low DNA quality since the samples were collected from 5 institutions and there might be deviation of FFPE tissue handling and collection.

Regarding clinical characteristics between the patients with and without $B R C A$ gene mutation, the present study did not find any significant differences in terms of age, disease, stage, primary outcome, platinum-sensitivity status, and survivals after treatment. Kuberlac et al., (2019) reported that their patients with both germline and somatic BRCA mutation had younger age and had significantly higher rate of complete pathologic response during the first and second platinum-sensitive relapse, and longer overall survival for advanced stage treated with neo-adjuvant chemotherapy when compared to 52 patients without $B R C A$ mutation. Although this study also found younger age among $B R C A$ mutation, the positive $B R C A$ gene did not serve as a favorable prognostic factor. The small number of patients in this study may limit any meaningful clinical summary regarding the prognostic role of $B R C A$ mutation.

In conclusion, tissue $B R C A 1 / 2$ mutation positive was presented in about $17 \%$ of high grade epithelial ovarian, fallopian, and peritoneal cancers. The most frequent histology was the serous cancer.

\section{Acknowledgements}

Thai Gynecologic Cancer Institute (TGCS) : for support this project

\section{Declarations}

Ethics approval

The study was approved by local ethic committees of each participating institution and had consent to participate.

Mahidol University: MURA2018/487

Chiang Mai University: OBG-12561-05448/ 200/2561

Chulalongkorn University: 959-2508

Navamindradhiraj University: COA 142/2561

Chulabhorn Hospital: 031-2561

\section{Funding}

This study was supported by Astra Zeneca Thailand which mostly provided the cost of genetic sequencing. However, the funders had no role in study design, data collection and analysis, decision to publish, or preparation of the manuscript.

\section{Authors'contributions}

P.S and A.L conceived of the presented idea. PS,AL,TM,TK,NK developed the theory and performed the computations. TC, SK, NP, ST and TL verified the analytical methods (Patho.) PS, CT, SS, NI, WC verified the analytical methods (LAB). ST, TM, AL, supervised the findings of this work for PS. All authors discussed the results and contributed to the final manuscript.

\section{Conflict of Interest Statement}

The authors have no conflicts of interest to disclose.

\section{References}

Cancer Genome Atlas Research Network (2011). Integrated genomic analyses of ovarian carcinoma. Nature, 474, 609-15.

Chao A, Chang TC, Lapke N, et al (2016). Prevalence and clinical significance of BRCA1/2 germline and somatic mutations in Taiwanese patients with ovarian cancer. Oncotarget, 7, 85529-41.

Dougherty BA, Lai Z, Hodgson DR, et al (2017). Biological and clinical evidence for somatic mutations in BRCA1 and BRCA2 as predictive markers for olaparib response in high-grade serous ovarian cancers in the maintenance setting. Oncotarget, 8, 43653-61.

da Cunha Colombo Bonadio RR, Fogace RN, Miranda VC, Diz MDPE (2018). Homologous recombination deficiency in ovarian cancer: a review of its epidemiology and management. Clinics (Sao Paulo), 73, e450s.

Einaga N, Yoshida A, Noda H, et al (2017). Assessment of the quality of DNA from various formalin-fixed paraffinembedded (FFPE) tissues and the use of this DNA for next-generation sequencing (NGS) with no artifactual mutation. PLoS One, 12, e0176280.

Enomoto T, Aoki D, Hattori K, et al (2019). The first Japanese nationwide multicenter study of BRCA mutation testing in ovarian cancer: CHARacterizing the cross-sectionaL approach to Ovarian cancer geneTic TEsting of BRCA (CHARLOTTE). Int J Gynecol Cancer, 29, 1043-9.

Goodheart MJ, Rose SL, Hattermann-Zogg M, et al (2009). BRCA2 alteration is important in clear cell carcinoma of the ovary. Clin Genet, 76, 161-7.

Gori S, Barberis M, Bella MA, et al (2019). Recommendations for the implementation of BRCA testing in ovarian cancer patients and their relatives. Crit Rev Oncol Hematol, 140, 67-72.

Hennessy BT, Timms KM, Carey MS, et al (2010). Somatic mutations in BRCA1 and BRCA2 could expand the number of patients that benefit from poly (ADP ribose) polymerase inhibitors in ovarian cancer. J Clin Oncol, 28, 3570-6.

Jansaka N, Suprasert P (2014). Survival outcomes of recurrent epithelial ovarian cancer: experience from a Thailand northern tertiary care center. Asian Pac J Cancer Prev, 15, 10837-40.

Kubelac P, Vlad C, Berindan Neagoe I, Irimie A, Achimas Cadariu P (2019). The clinical features associated with mutated BRCA1 and 2 genes in ovarian cancer 
patients. $J$ BUON, 24, 1538-43.

Kwon BS, Byun JM, Lee HJ, et al (2019). Clinical and genetic characteristics of BRCA1/2 mutation in Korean ovarian cancer patients: A multicenter study and literature review. Cancer Res Treat, 51, 941-50.

Konstantinopoulos PA, Norquist B, Lacchetti C, et al (2020). Germline and somatic tumor testing in epithelial ovarian cancer: ASCO Guideline. J Clin Oncol, 38, 1222-45.

Ledermann J, Harter P, Gourley C, et al (2014). Olaparib maintenance therapy in patients with platinum-sensitive relapsed serous ovarian cancer: a preplanned retrospective analysis of outcomes by BRCA status in a randomised phase 2 trial. Lancet Oncol, 15, 852-61.

Li MM, Datto M, Duncavage EJ, et al (2017). Standards and guidelines for the interpretation and reporting of sequence variants in cancer: A Joint Consensus Recommendation of the Association for Molecular Pathology, American Society of Clinical Oncology, and College of American Pathologists. J Mol Diagn, 19, 4-23.

Manchana T, Phoolcharoen N, Tantbirojn P (2019). BRCA mutation in high grade epithelial ovarian cancers. Gynecol Oncol Rep, 29, 102-5.

Manchana T, Phowthongkum P, Teerapakpinyo C (2019). Germline mutations in Thai patients with nonmucinous epithelial ovarian cancer. World J Clin Oncol, 10, 358-68.

Pujade-Lauraine E, Ledermann JA, Selle F, et al (2017). Olaparib tablets as maintenance therapy in patients with platinum-sensitive, relapsed ovarian cancer and a BRCA1/2 mutation (SOLO2/ENGOT-Ov21): a double-blind, randomised, placebo-controlled, phase 3 trial. Lancet Oncol, 18, 1274-84.

Richards S, Aziz N, Bale S, et al (2015). Standards and guidelines for the interpretation of sequence variants: a joint consensus recommendation of the American College of Medical Genetics and Genomics and the Association for Molecular Pathology. Genet Med, 17, 405-24.

Torre LA, Trabert B, DeSantis CE, et al (2018). Ovarian cancer statistics, 2018. CA Cancer J Clin, 68, 284-96.

Vergote I, Banerjee S Gerdes AM, van Asperen C, et al (2016). Current perspectives on recommendations for BRCA genetic testing in ovarian cancer patients. Eur J Cancer, 69, 127-34.

Wilailak S, Lertchaipattanakul N (2016). The epidemiologic status of gynecologic cancer in Thailand. J Gynecol Oncol, 27, e65.

Webber K, Friedlander M (2017). Chemotherapy for epithelial ovarian, fallopian tube and primary peritoneal cancer. Best Pract Res Clin Obstet Gynaecol, 41, 126-38.

Wu X, Wu L, Kong B, et al (2017). The first nationwide multicenter prevalence study of germline BRCA1 and BRCA2 mutations in Chinese ovarian cancer patients. Int $J$ Gynecol Cancer, 27, 1650-7.

Zhao Q, Yang J, Li L, et al (2017). Germline and somatic mutations in homologous recombination genes among Chinese ovarian cancer patients detected using next-generation sequencing. J Gynecol Oncol, 28, e39.

\section{(ब) $(\otimes$}

This work is licensed under a Creative Commons AttributionNon Commercial 4.0 International License. 